\title{
Research Paper \\ Reasons for Nursing Home Placement of Older Women in Tabriz, Iran: A Content Analysis
}

\section{Mehdi Abbasian ${ }^{1} \odot$, Mehdi Nakhodaeezah², Shamsedin Namjoo ${ }^{2} \odot$, Zahra Khalili ${ }^{3}$, Leila Jahangiry ${ }^{4}$, Reza Fadayevatan $^{2,5} \odot$, *Hossein Matlabi ${ }^{4}$}

1. Tabriz Health Services Management Research Center, Tabriz University of Medical Sciences, Tabriz, Iran.

2. Department of Ageing, University of Social Welfare and Rehabilitation Sciences, Tehran, Iran.

3. Department of Medical Surgical Nursing, School of Nursing and Midwifery, Ardabil University of Medical Sciences, Ardabil, Iran.

4. Department of Health Education and Promotion, Faculty of Health Sciences, Tabriz University of Medical Sciences, Tabriz, Iran

5. Iranian Research Center on Aging, University of Social Welfare and Rehabilitation Sciences, Tehran, Iran.

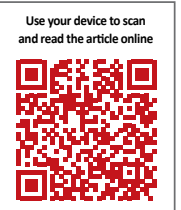

Citation Abbasian M, Nakhodaeezah M, Namjoo Sh, Khalili Z, Jahangiry L, Fadayevatan R, et al. [Reasons for Nursing Home Placement of Older Women in Tabriz, Iran: A Content Analysis (Persian)]. Iranian Journal of Ageing. 2019; 13(4):406-417. https:// doi.org/10.32598/SIJA.13.4.406

https://doi.org/10.32598/SIJA.13.4.406

Keywords:

Elderly, Nursing homes, Qualitative research, Tabriz, Content analysis

\section{A B STRACT}

Objectives In consistent with the growing rate of elderly population, the burden of chronic diseases has dramatically increased, and subsequently, the need for long-term care has increased, too. The population rate of elderly women is higher than men, and their admission rate to nursing homes is more than men. The present study explained different reasons for the admission of the elderly women to nursing homes.

Methods \& Materials In total, 13 participants were recruited through purposive sampling method. Semistructured face-to-face interviews were applied in this qualitative research. Content analysis was used for data analysis.

Results Two key factors were the causes of elderly women living in nursing homes. These factors included 'underlying reasons' and 'situational reasons'. The obtained results indicate that the situational reasons played a dominant role in choosing nursing home care. Older women noted several barriers for the lack of participation in the community and at home. Such factors included financial problems, unwanted marriage, abuse, and neglect. Moreover, falls, accidents, loneliness, and emotional problems were classified as major situational reasons.

Conclusion In consistent with the growing rate of elderly women, their burden of care continues to rise. Moreover, elderly abuse will increase in this population due to declining financial resources. This could lead to the sending the elderly people to nursing homes. To prevent these factors and facilitate conditions for families and elderly people to live in their desired place, providing community-based services can be useful.

\section{Extended Abstract \\ likely to be sent to nursing homes compared to older men \\ 1. Objectives

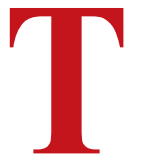 \\ he burden of chronic diseases increases with age, which subsequently calls for long-term care [1]. In most parts of the world, the aged women population is higher than that of men, and they are more \\ [2]. Moving to residential care homes has been reported as one of the hardest experiences of the elderly and their caregivers. Such transition can cause different degrees of depression and loss of social support in the elderly [3]. \\ Nursing home is the most important residential area for some of the elderly groups. However, it is generally nec- essary to recognize the reason for choosing to live in these \\ * Corresponding Author \\ Hossein Matlabi, PhD. \\ Address: Department of Health Education and Promotion, Faculty of Health Sciences, Tabriz University of Medical Sciences, Tabriz, Iran. \\ Tel: +98 (411) 3357580 \\ E-mail: hm1349@gmail.com}


care homes. By this knowledge, we can use preventive and treatment methods and services to help the elderly women stay the longest possible in their own houses.

\section{Methods and Materials}

This qualitative research used content analysis approach. Samples were selected using purposeful sampling method among the elderly women living in nursing homes in Tabriz City, Iran. The study data were performed through semi-structured and face-to-face interview with the participants. Inclusion criteria were being $\geq 60$ years old, having cognitive abilities according to a short cognitive test result, willingness to express attitudes about the research objective, and the having able to speak and participate in interviews. Those with cognitive problems and related diseases and younger than 60 years were excluded from the study.

The process of sample selection continued until the saturation of data. Eventually, 13 elderly women aged 60-91 years were selected. Duration of their stay in nursing homes ranged from 5 months to 10 years. Required descriptions of the goals and importance of the research were provided to the study participants. After obtaining consent forms, the time and place of the interview was determined by the subjects. The interview environment was comfortable enough for recording a high quality sound. The participants were assured of the confidentiality of their information. They were able to withdraw from the study as desired. This study was approved by the Ethics Committee of Tabriz University of Medical Sciences.

\section{Results}

Two main concepts were identified as key factors associated with the causes of elderly women living in nursing homes; "underlying factors" and "situational factors". Elderly women reported several problems that deprived them of participation in the community and home chores. Such factors included financial problems, unwanted marriage, inconvenience, negligence, falls, accidents, illness, loneliness, and emotional problems.

Age, gender, career, and income level were considered as the demographic characteristics. Despite having different lifestyles, these factors almost had the same effect on the subjects. Considering these factors, the elderly prefer to live in nursing homes which can help reduce their stress. Family factors were related to the needs and conditions of the elderly in recent years. Living conditions and the unfulfilled needs of the elderly women through many years were the causes of staying in nursing homes. In other words, such conditions were the facilitators of living in nursing homes. But appropriate living conditions, family/children and even grandchildren support, a dynamic lifestyle, feeling of usefulness, self-care ability, lack of loneliness, attachment style, etc. were reasons for staying at their own homes.

This can increase their life satisfaction and their quality of life. On the other hand, lack of the aforementioned conditions along with unwanted marriage, inconvenience, and negligence were the reasons for staying in nursing homes. According to the obtained data, the subcategories of accidents, falls, and acute illnesses were extracted from the "accidental" dimension. In this regard, the most reported causes were the lack of caregiver and inability to self-care. Under "imposed" dimension, some subjects reported enforced factors and decisiveness as their reasons for living in nursing homes.

Enforced living in nursing homes was among the factors affecting their depression. With respect to "voluntary" dimension, the benefits of living in nursing homes were reported. Elderly women are transferred to nursing homes after years of living with family, friends or relatives. Soon after feeling lonely or experiencing emotional problems, the lack of partner or security, the lack of caregiver, inability to cook, self-care inability, etc., they were admitted to nursing homes. Such places can improve their physical conditions and their quality of life.

\section{Conclusion}

Lack of support, bio-psychological factors, low literacy and celibacy were among the main reasons of elderly women in Tabriz to live in nursing homes. It is necessary to empower the elderly in care centers and achieve the most possible healthy aging and maintaining the elderly's contact with community. Thus, further studies on social factors and those introduced in this research at a national level and in different cultural contexts seems necessary. It also seems that the services needed to accommodate the elderly woman in the current and desired houses (such as home care services at different levels, supportive and social services, and companionship services) should be integrated with the health system.

\section{Ethical Considerations}

\section{Compliance with ethical guidelines}

This paper has been approved by Tabriz University of Medical University. The research goal and process 
were explained to the participants and they were assured of the confidentiality of their information. They were free to quit the study at any time.

\section{Funding}

This research did not receive any specific grant from funding agencies in the public, commercial, or not-forprofit sectors.

\section{Authors contributions}

Conceptualization, validation: Hossein Matlabi, Mehdi Abbasian, and Mehdi Nakhodaeezah; Methodology: Reza Fadayevatan and Hossein Matlabi; Formal analysis: Hosein Matlabi and Mehdi Abbasian; Investigation, resources, and draft preparation: Mehdi Abbasian, Shamsedin Namjoo, and Mehdi Nakhodaeezah; Editing: Hossein Matlabi; Visualization, supervision, and project administration: Hossein Matlabi and Reza Fadayevatan; and Funding acquisition: Hossein Matlabi and Mehdi Abbasian.

\section{Conflict of interest}

The authors declared no conflict of interest.

\section{Acknowledgements}

Authors would like to thank authorities and personnel of the study nursing home for their valuable cooperation. 


\section{علل اقامت زنان در سراى سالمندان شهر تبريز: تحليل محتوا}

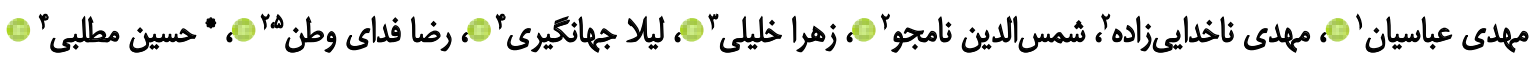

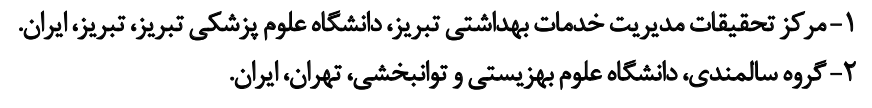

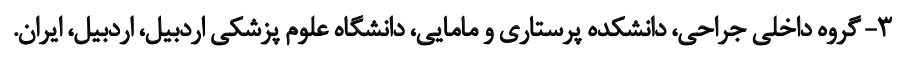

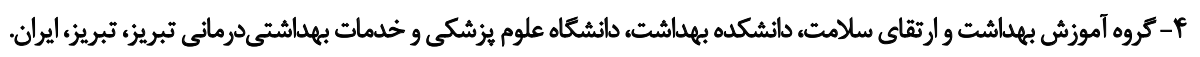

ه- مركز تحققات سالمندى، دانشعاه علوم بهزيستى و توانبخشى، تهران، ايران.

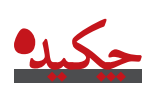

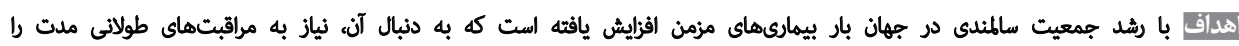

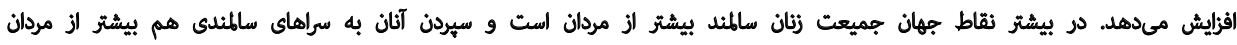

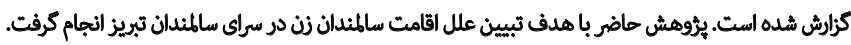

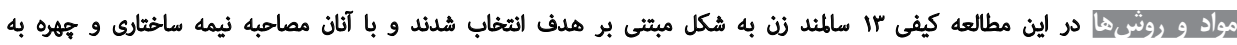

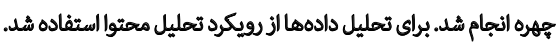

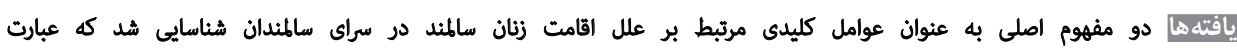

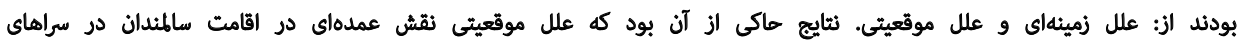

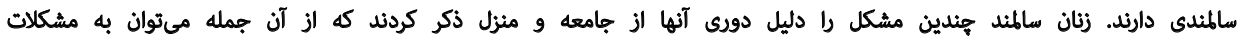

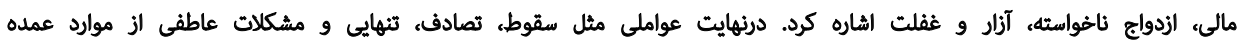

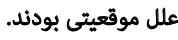

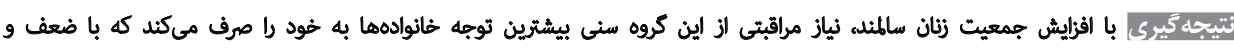

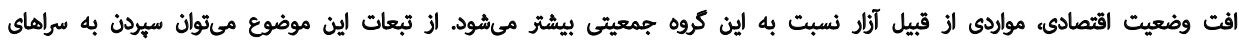

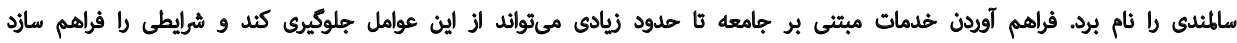
كه سالمندان در محيط دلخواه خود زئدكى كنين.

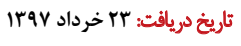

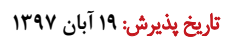

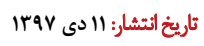

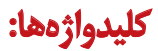

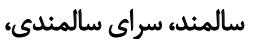

مطالعه كيفى، تبريز،

تحليل محتوا

به علت علاقه سالمئدان به حفظ استقلال در انجام فعاليتهاى

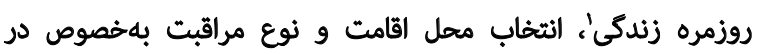

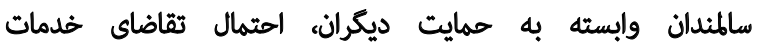

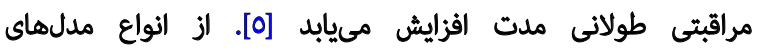

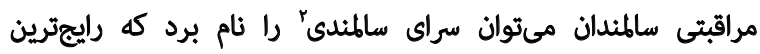

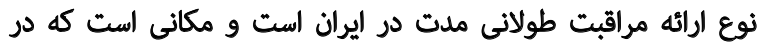

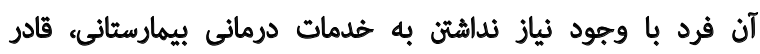

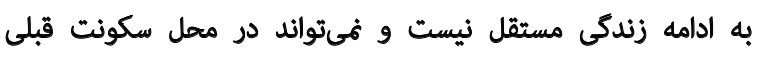

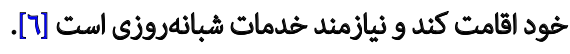

1. Activity of daily living 2. Nursing home

جمعيت دنيا و ايران بهسرعت در حال سالمند شدن است.

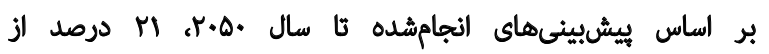

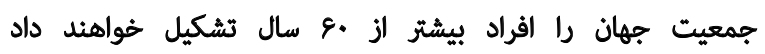

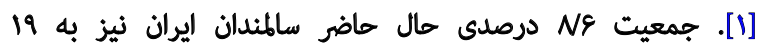

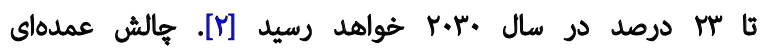

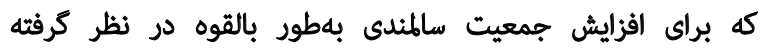

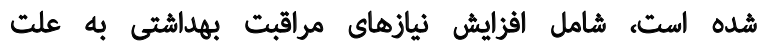

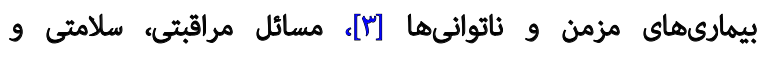

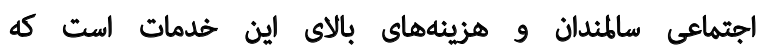

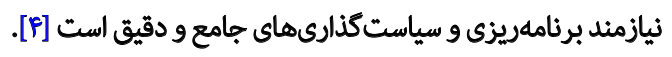

$$
\text { … }
$$

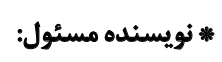

دكتر حسين مطلبى مئول

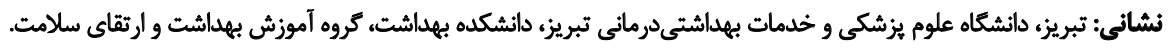
تلفن: يست الكترونيكي: بلون 
در محل سكونت قبلى هستثد: جايى كه سالمثد به آن تعلق

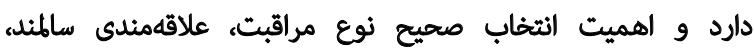

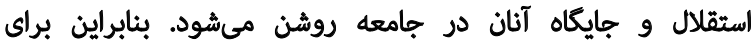

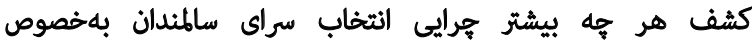

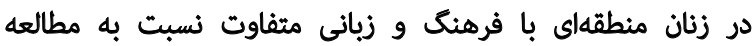

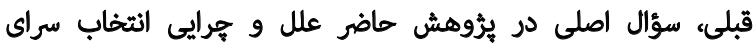

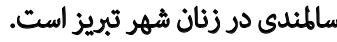

$$
\text { روش مطالعه }
$$

اين مطالعه به روش تحقيق كيفى با رويكرد تحليل محتواى

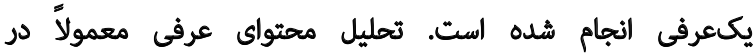

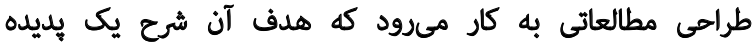

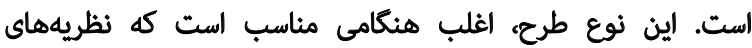

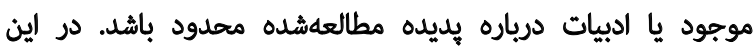

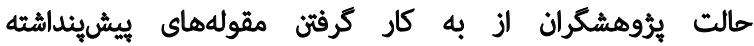

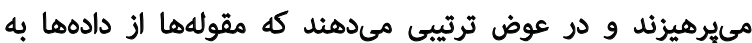

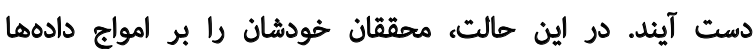

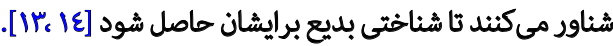

علل اقامت سالمندان زن در سراهاى سالمئدى در مناطق تئق

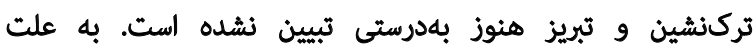

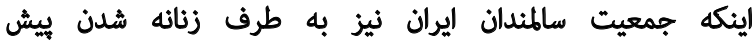

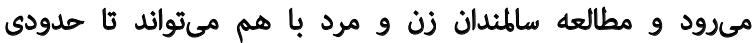

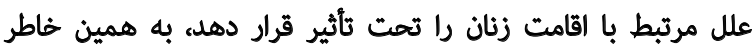

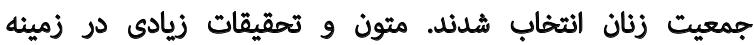

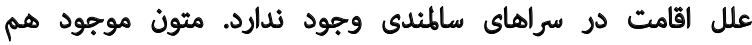

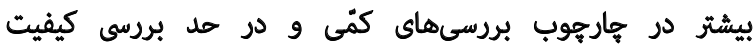

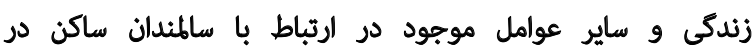

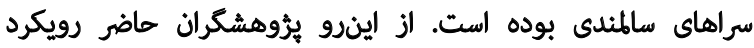

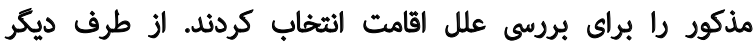

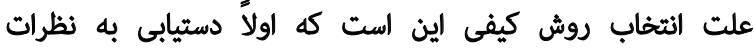

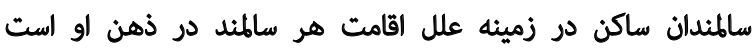

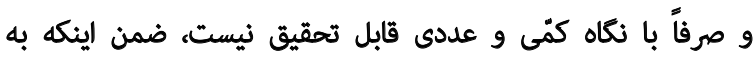

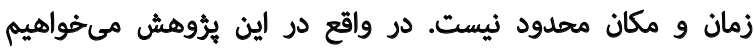

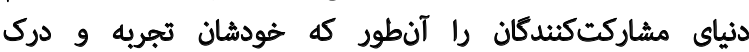

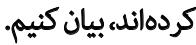

در اين بُروهش با توجه به هدف مطالعه، شركتكندكان بر

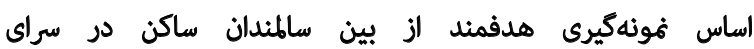

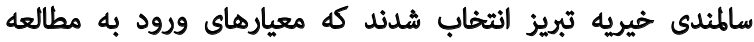

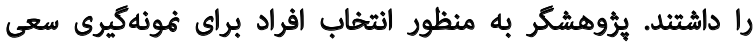

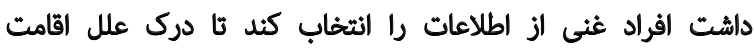

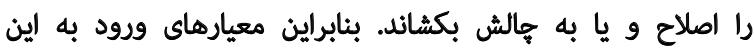

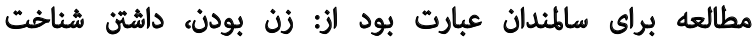
كه با بررسى آزمون كوثاه شناختى مشخص شده تمايل بهان به بيان

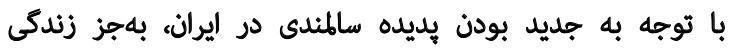

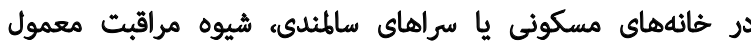

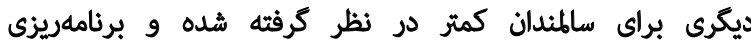

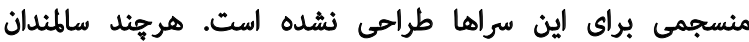

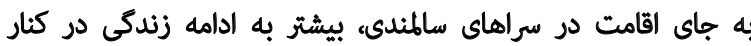

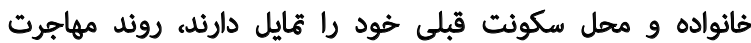

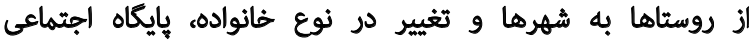

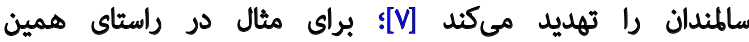

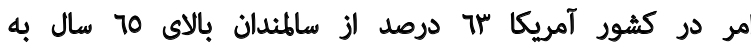

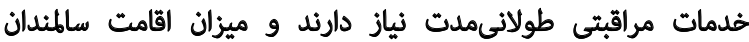

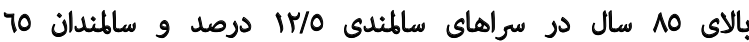

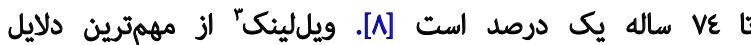

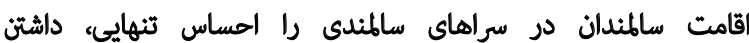

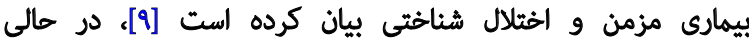
كه سالاروند و همكاران علاوه بر آنهاء وضعيت اقتصادي ضعيف، كردان

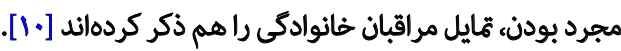

انثقال به مراكز خدمات سالمندى به عنوان يكى از سخترين

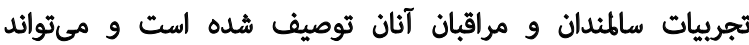
درجات متفاوتى از الحساس طرد شدن، افسردكى و و از دمانت

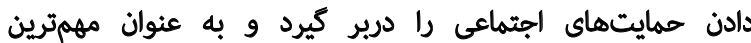

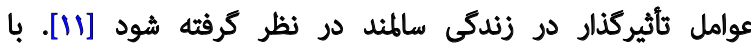

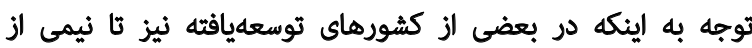

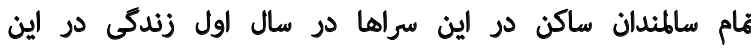

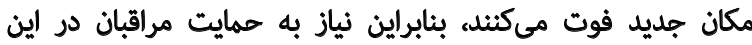

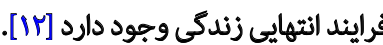

تغيير نوع خانواده ايرانى از كسترده به هستهاي، كاهش

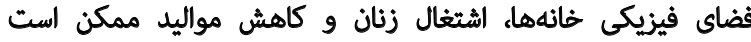

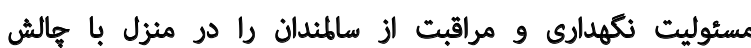

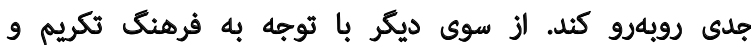

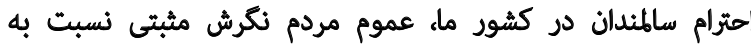

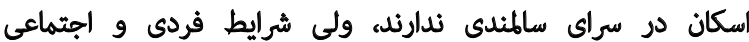

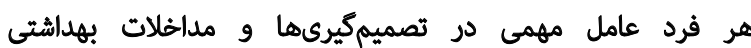

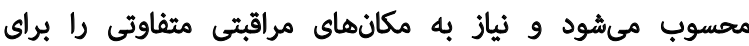
سالمئدان هر جامعه بيان مىكند [•]"] به نظر مىرسد هرايى انتخاب سراهاى سالمندى توسط هر فرد

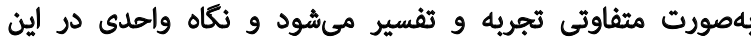

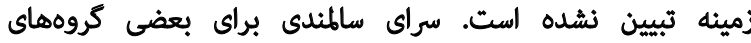

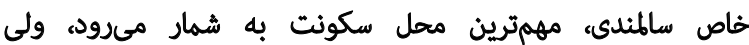

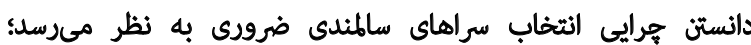

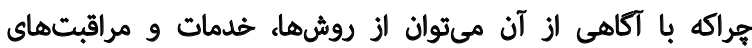

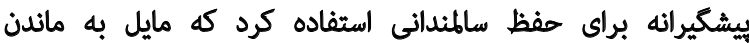


موضوعى بزركترى قرار داد ثا به مفاهيم اصلى دست ييدا كند.

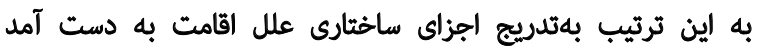

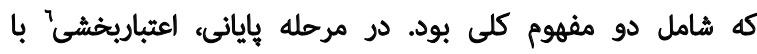
ارجاع به هر ثمونه ويرسيدن درباره يافتهها انجام كرفت. در اين يُووهش براى استحكام تهقيق از دو معيار اطمينانيذيرى

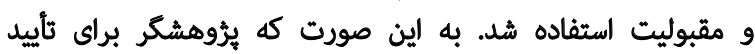

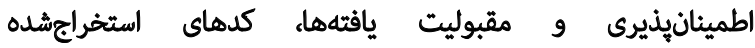
را به شركتكنيدكان ارجاع داد و و با تأييد آنان، يافتهها معتبريت

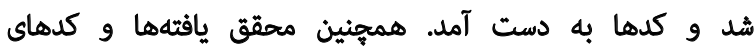

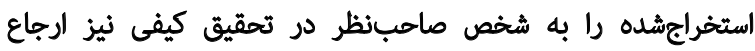

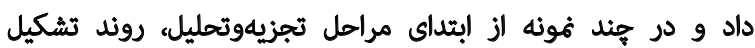

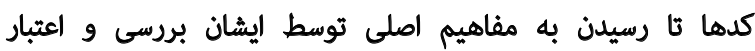
يافتهاى تهقيق از نظر ايشان تأييد شد.

يس از انتخاب شركتكندكان بر اساس معيارهاى ورود به

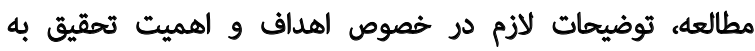

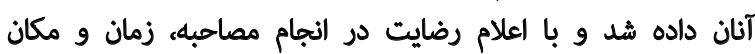

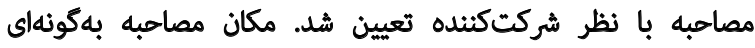

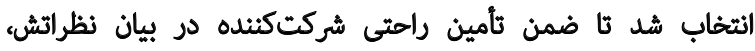

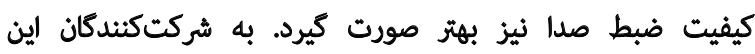

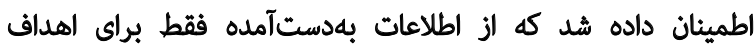

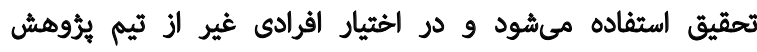

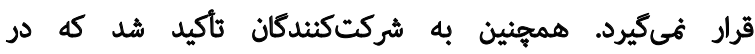

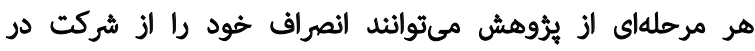

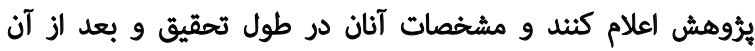

به مورت محرمانه هفظ مي شونود.

\section{يافتهها}

در مجموع از "Iا مشاركتكننده زن، M M مصاحبه به عمل آمد.

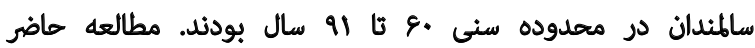

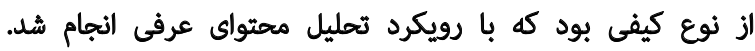

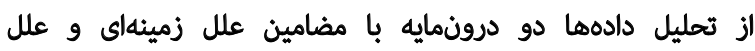

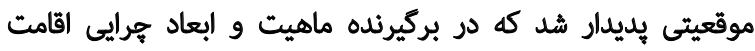

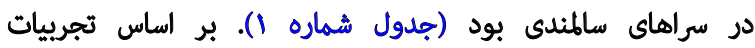

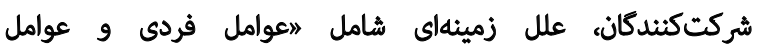

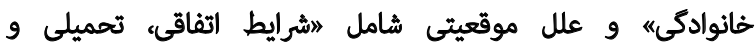

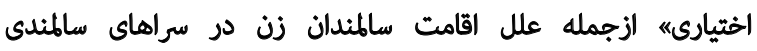

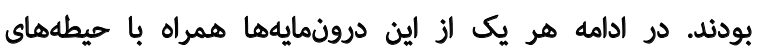

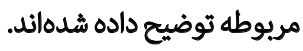

$$
\text { علال زميئهاى }
$$

با توجه به سؤالات مطرحشده، سالمندان زن علل زمينهاى
و اظهار درونى خود نسبت به مفهوم و موضوع مورد تمقيق،

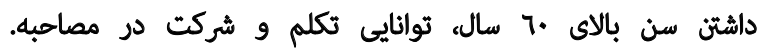

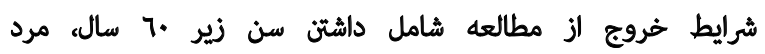
بودن، داشتين مشكل شناختى و بيمارىهاى مرتبط بودي.

فرايند انتخاب نمونهها تا اشباع دادهها ادامه يافت؛ يعنى تأن

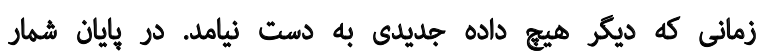

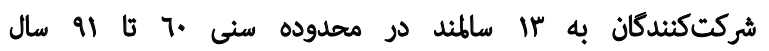

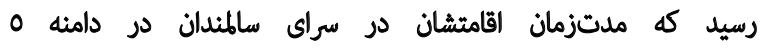

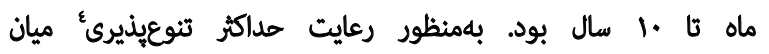

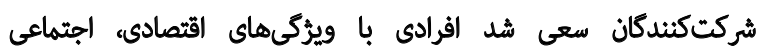

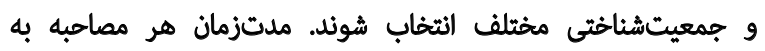

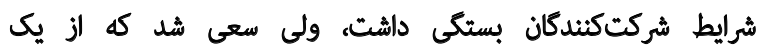

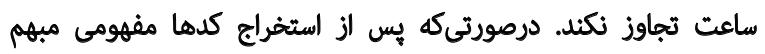
بود، مصاحبه ديكرى برايى روشنسازى انجام مىكرفت.

يس از بررسى و ثبت اطلاعات جمعيتشناختى مشاركتكننده،

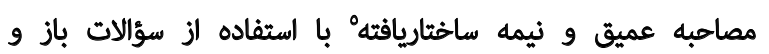

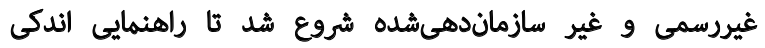

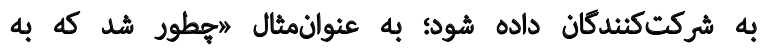

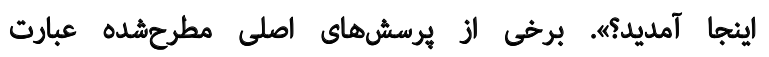

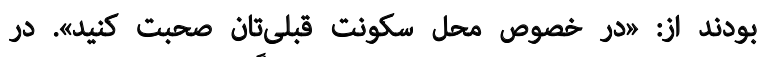

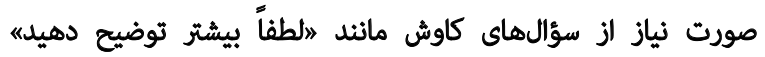

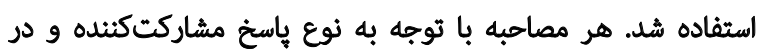

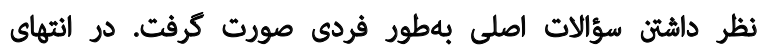

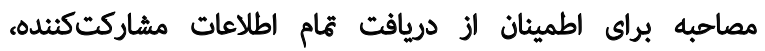

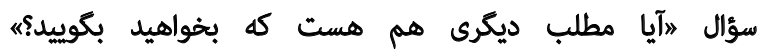

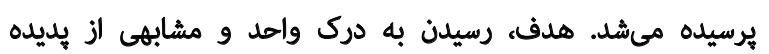

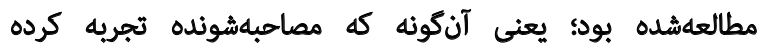

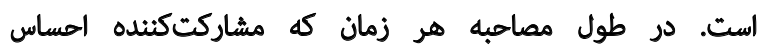

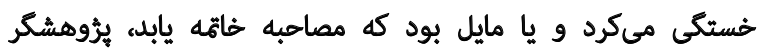

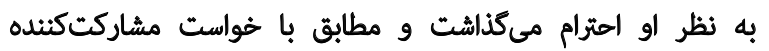
عمل مئشي. تمام مصاحبههاى انجامشده با ضبطصوت ضبط شيط شدند. جهندين

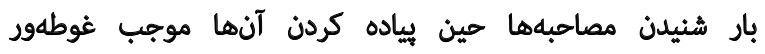

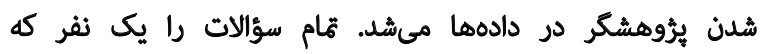

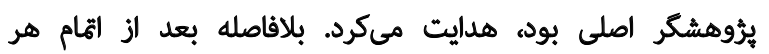

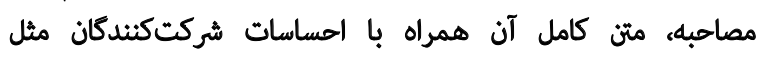

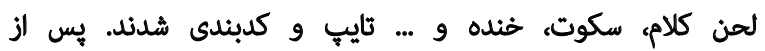

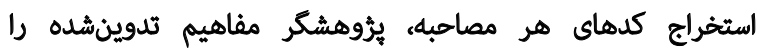

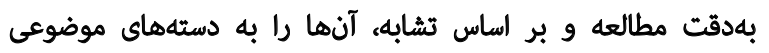

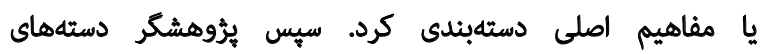

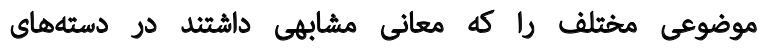

4. Maximum variation

5. Semi structural interview 
همين نه براى اون خواهرم خواستكارى اومد و نه كذاشتند من

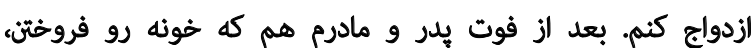

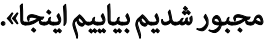

يكى ديكر از سالمندان در اينباره كفت: "به خاطر مرك

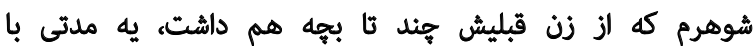

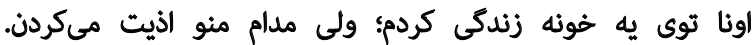

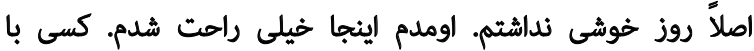

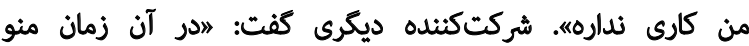

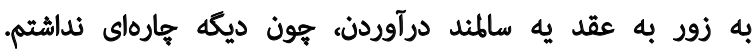

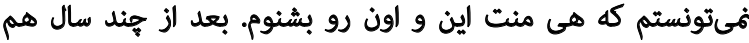

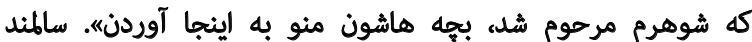

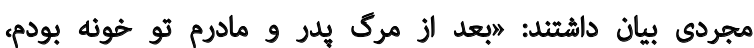

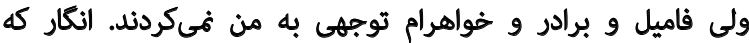

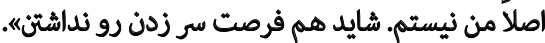

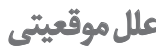

يك علت مهم و اساسى ديكر در اقامت شهروندان ارشد در

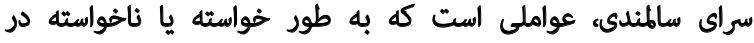
سالهاى اخير بر زندكى فرد اثركذار بوده و زئدكى او خوائ را دكركون

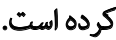

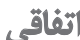

با توجه به دادهها، الز اين طبقه، زيرطبقات سانصه، سقوط،

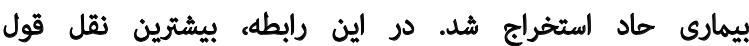

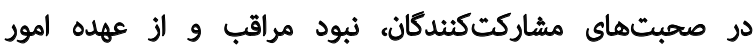

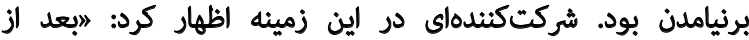

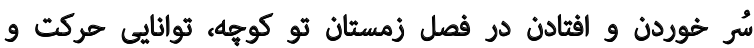

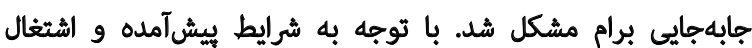

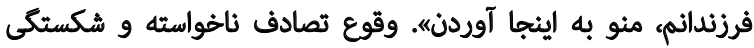

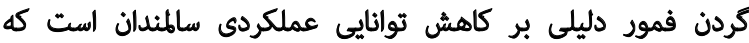

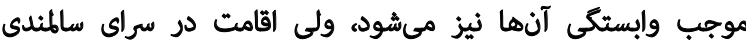

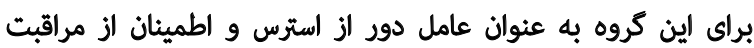

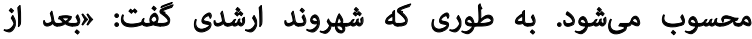

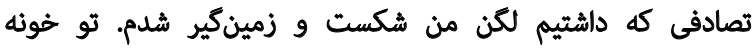

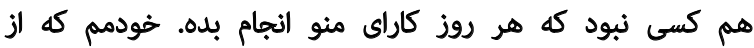

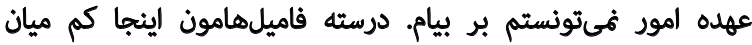

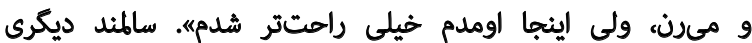

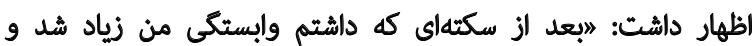

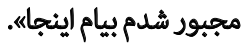

تحميلى

تعدادى از سالمثدان عوامل اجبارى و فريب را دلايل سكونت

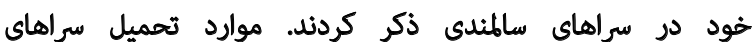

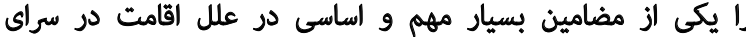

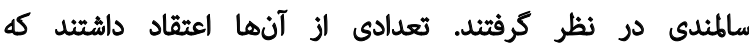

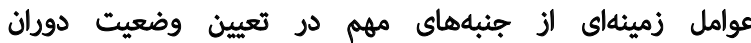
سالمندى است كه سالمندان را مستعد مراقبت و توجه مىكنداي

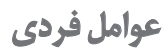

مشاركتكنيدكان در اين بيزوهش عوامل مربوط به سنه

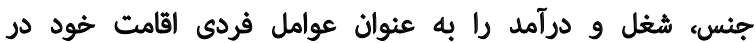

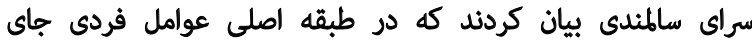

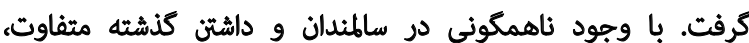
آنها تقرييا رويكرد مشابهى داشتئد. با اتخاذ اينرويكرد، سالمند اولويت را به سراى سالمندى مىدهد و و در آن صورت آئن اين ديدكاه

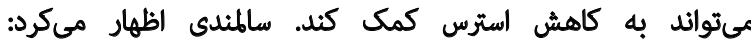

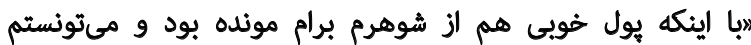

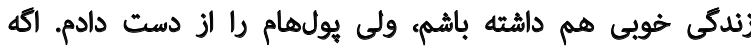

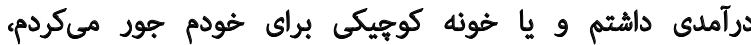

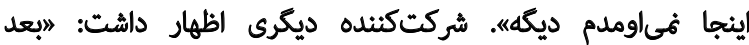

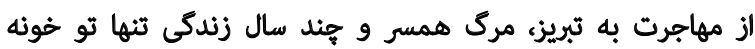

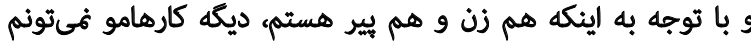
خودم انجام بلدم و اينجا راهت هترمه.

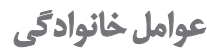
كاين طبقه مريوط به شرايط و نيازهاى سالمند در سالهاى

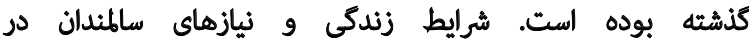

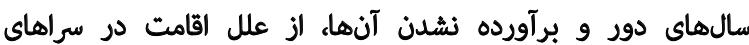

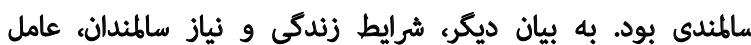

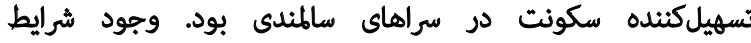

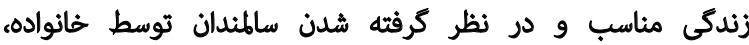

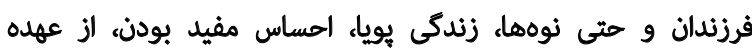

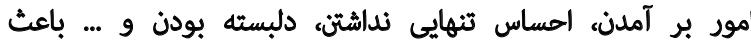

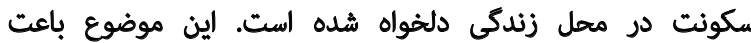

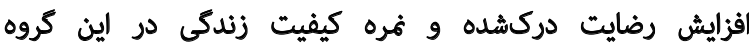

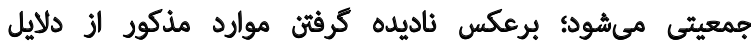

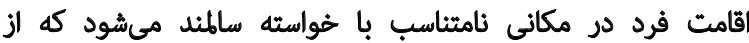
آن جمله ميتوان به الزدواج ناخواسته آلزار و غفلت اشاره كردا. شركتكنندهاي بيان كرد: البه خاطر اينكه بيسواد بودمه بعد

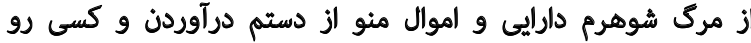

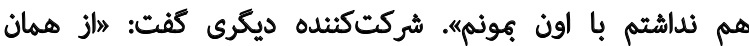

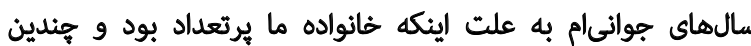

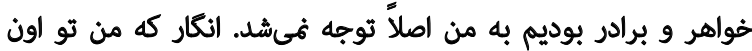

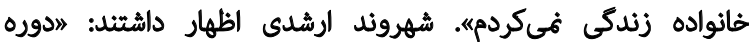

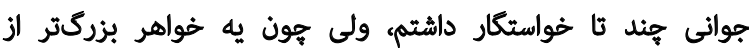

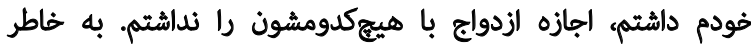


جدول ا. خلاصهاى از علل اقامت در سراى سالمثدان و جكونكىى رسيدن به مفاهيم اصلى

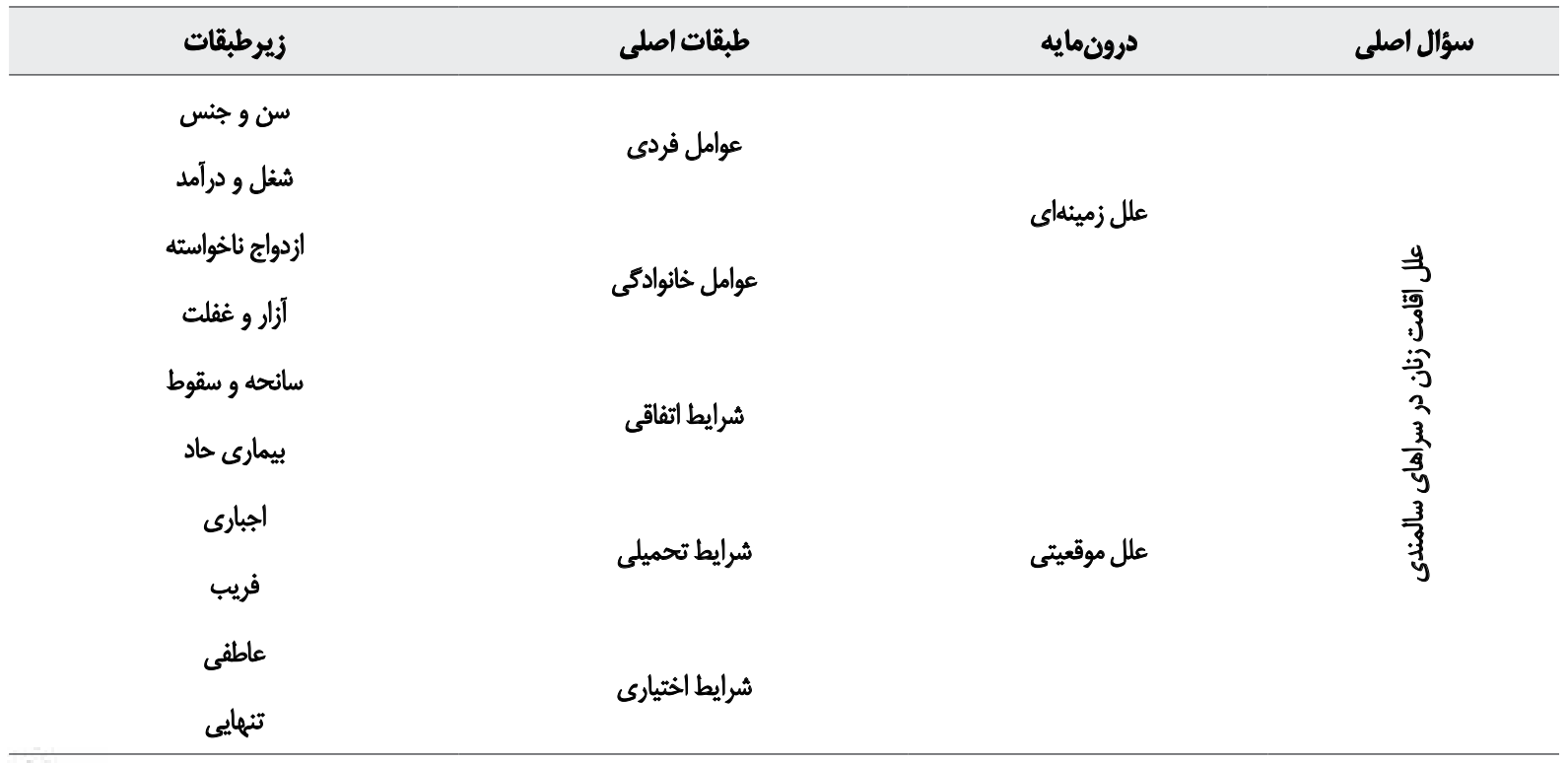

خيلى اذيت مىشدم و ديكه حس زندكى نداشتم. الز وقتى اومدم اينجا دوستانى ييدا كردم. اينجا به ما خوب مىرسند. خويهه.

$\stackrel{4}{*}$

هدف مطالعه حاضر دست يافتن به علل اقامت سالمندان زن در برد

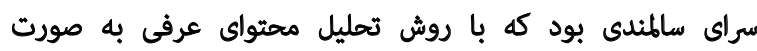

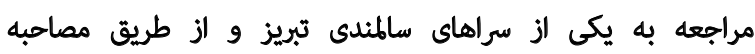

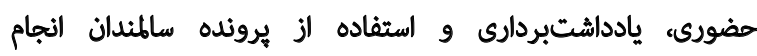

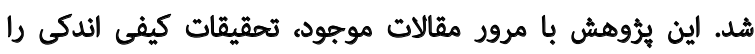

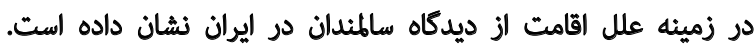

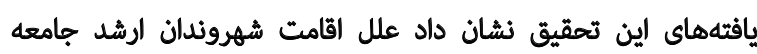

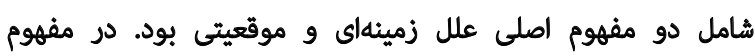

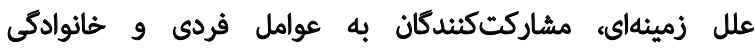

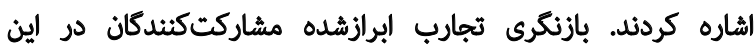

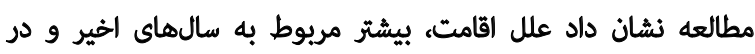

$$
\text { مفهوم علل موقعيتى بود. }
$$

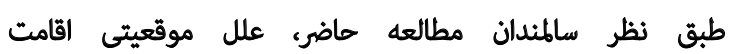

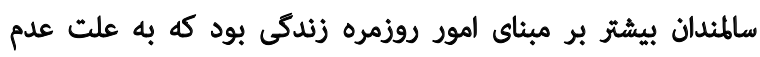

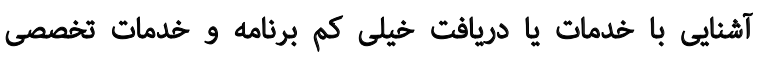

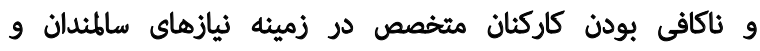

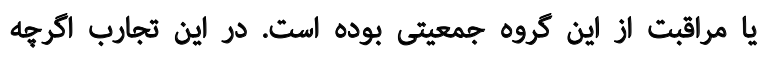

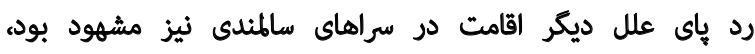

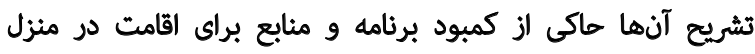

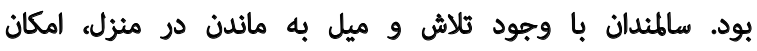
جهنين جيزى رانداشتند.

در سالهاى اخير سالمندان بيشتر از حمايتهاى غيرسمىى

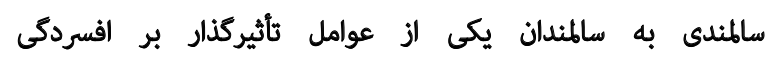
آنهاست. مشاركتكنيدكان نظرهاي خوني

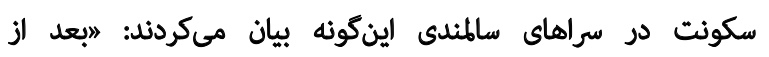

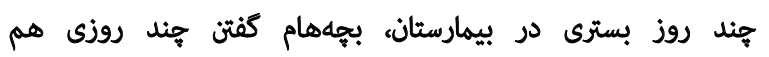

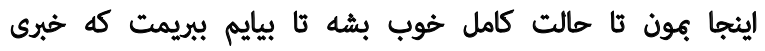

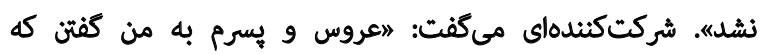

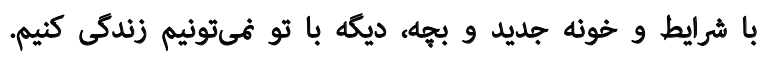
برى خونه سالمندان راحت ترىىه.

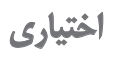

از مزيتهاي وجود سراى سالمندى مىتوان به مواردى اشاره

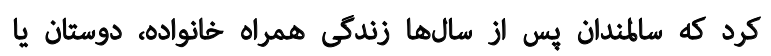

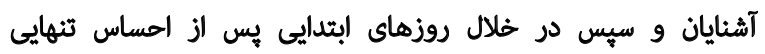

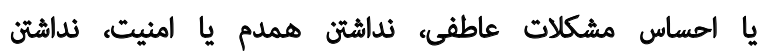

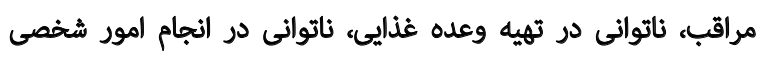

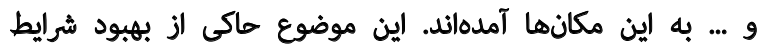

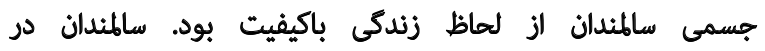

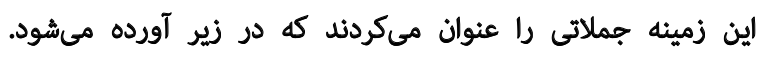

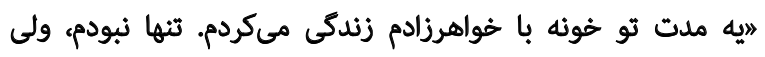

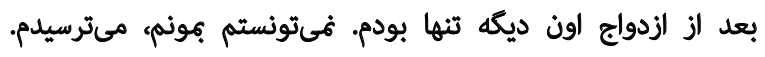

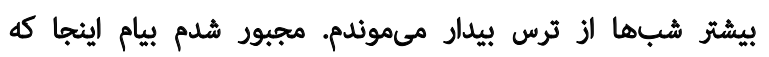
حداقل امنيت داشته باشمه. مشاركتكننده ديكرى مىكفت: "ابعد از مرك همسرم ديكه

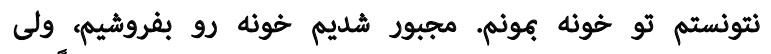

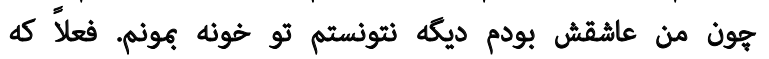

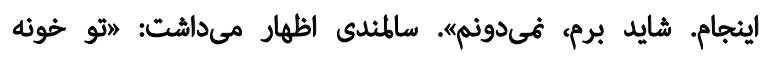




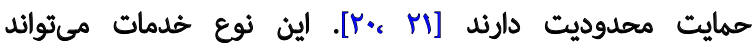

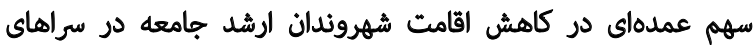

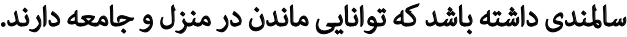

نداشتن حمايت در مفهوم علل موقعيتى ذكر شد و به دنبال

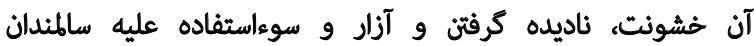

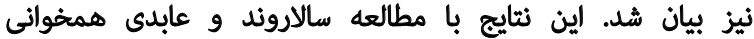
دارد. سالاروند و عابدى هم حمايت نداشتن سالمندان رابن را الز دلايل

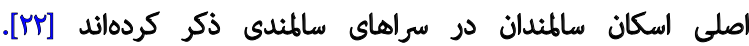

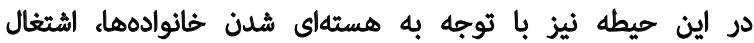

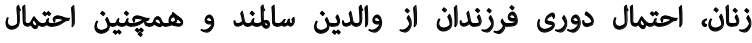

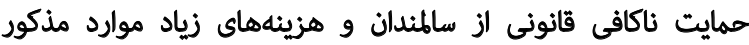

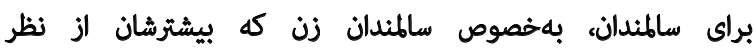
مالى وابسته هستند حمايتها كم خواهد شد.

در اين يُوهش مشاركتكنندكان به اهميث علل زمينهاى و دور

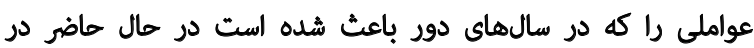

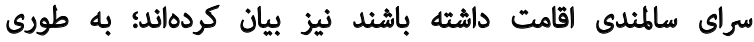

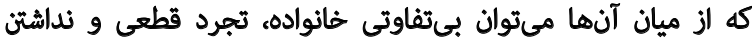

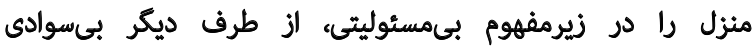

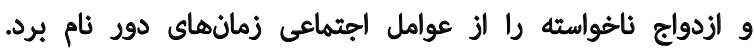

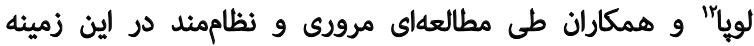

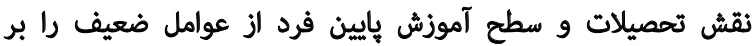

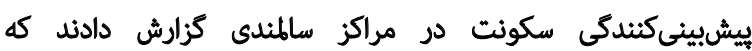

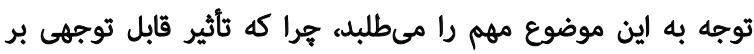

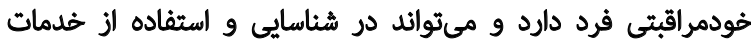

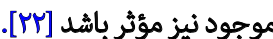
در مطالعات مختلف علل اقامت سالمندان در سراهاى سالمندى

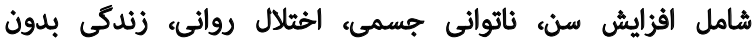

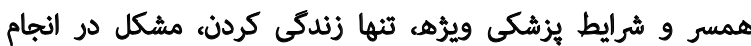

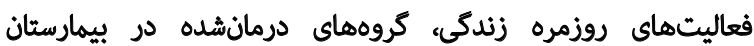

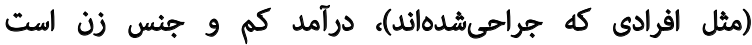

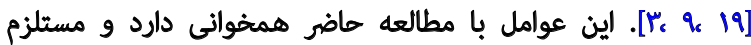
در اولويت قرار دادن نيازهاى سالمندان در كنار ساير كروههاي

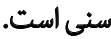

\section{نتيجلئيرى نهاييى}

با توجه به يافتهاى مطالعات ائجامشده و يروهش حاضر،

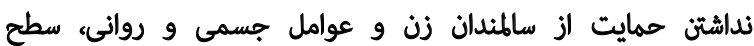

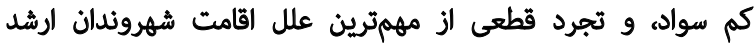

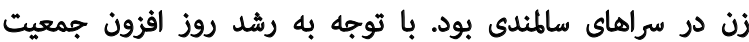

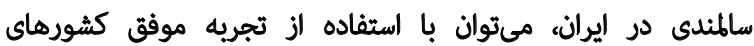
ييشرو در امر سالمندى و استفاده الز تجارب خانئ خدمات متنوع
خانواده، قوموخويش، همسايكان و خيّران براى ماندن در منزل

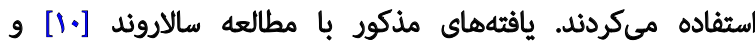

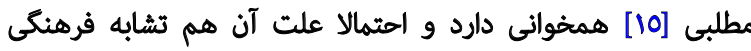

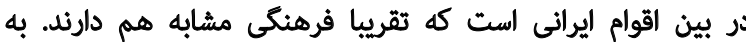

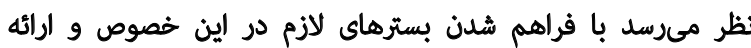

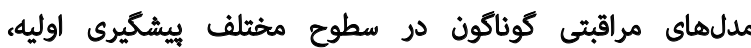

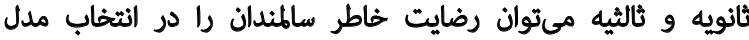
مراقبتى دلخواه و مناسب شرايط سالمند فراهم كرد. براى كم كردن اين مشكل در اغلب كشورهاى ييشرو در

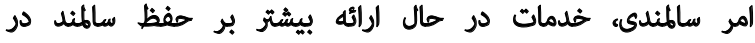

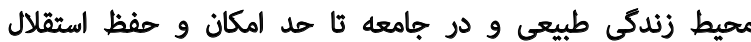

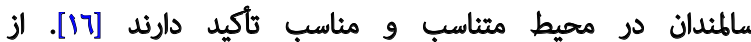

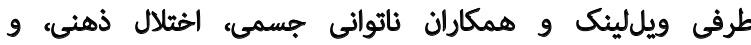

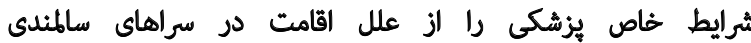

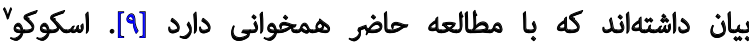

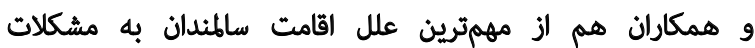

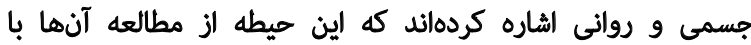

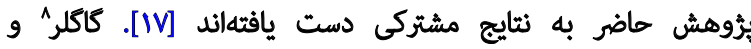

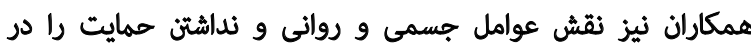
يذيرش در سراهاى سالمندى بهوضوح نشان دادهاند [1M] دركل بررسى عوامل مؤثر در اقامت سالمندان در سراهاى

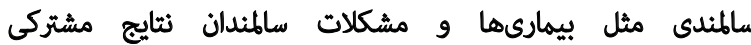

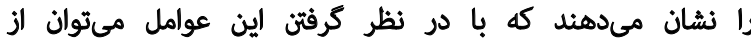

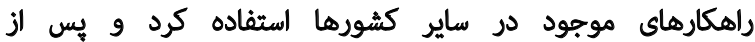

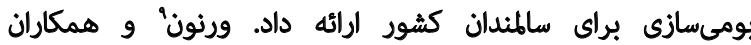

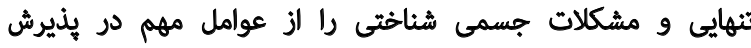

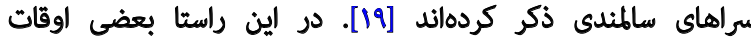

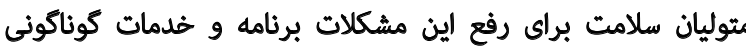

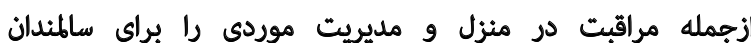

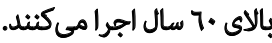

مراقبت منزل و مديريت موردى اشكال مخثلفى از كمك فردى

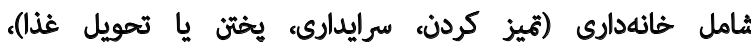

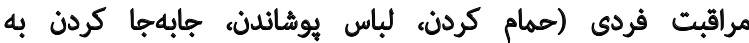

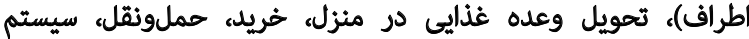

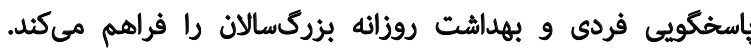

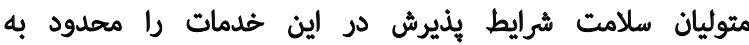

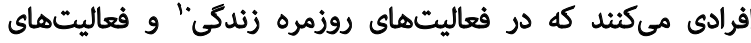

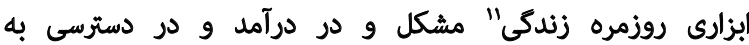

\section{Scocco}

8. Gaugler

9. Vernon

10. Activity of Daily Living (ADL)

11. Instrumental Activity of Daily Living (IADL) 
تعريفشده آنها براى سالمندان استفاده كرد نا سالمندانى فعال تعارض منأق

بنابر اظهار نويسندكان، اين مقاله تعارض منافع ندارد.

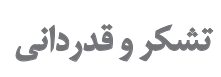

به منظور توانهثلسازى سالمثدان در مراكز مراقبتى و رسيدن

بلدينوسيله الز مسنولان و كاركنان سماى سالمندان كه همكارى لازم را با ئزوهشكران ابراز كردند، تشكر و قدردانى مى كنيم.

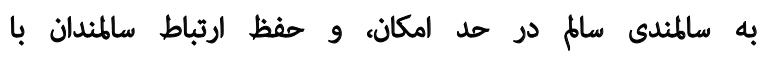
جامعه، بيشنهاد مىشود علاوه بر مطالعات رايج كه بيشتئر بُعد إندان يزشكى و شناختى را بررسى مىكند، تمقيقات بيشترى در زماد زمينه

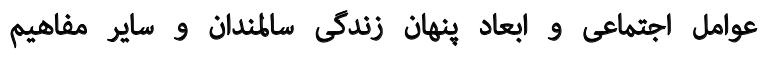

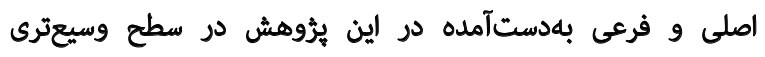

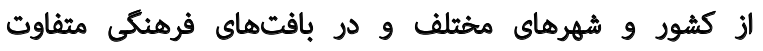

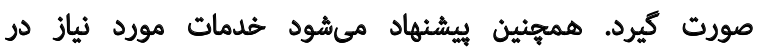

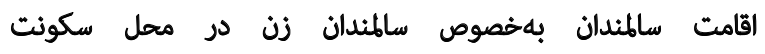
جارى و دلخواه كه در مطالعات مختلف به دست آمده است دمات درن

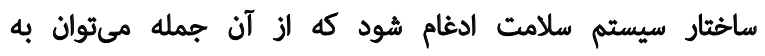

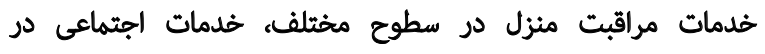

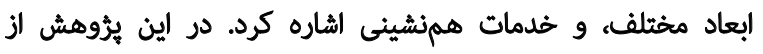

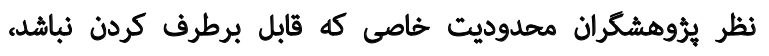

وجود نداشت.

مالاحظات اخلاقى - اتى

\section{بيروى ائز اصول اخلاق ثيؤهش}

دانشكاه علوم بزشكى تبريز اين تحقيق را ثأييد كرده است.

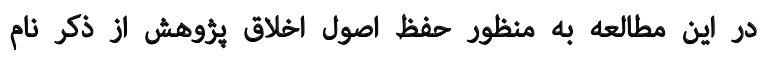

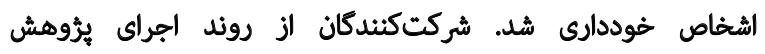

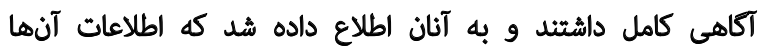

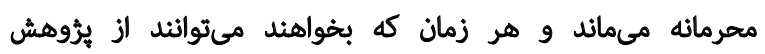
خارج شوند. مامب مالى اين يُروهش حامى مالى نداشته است. مشاركت نويسند مكان

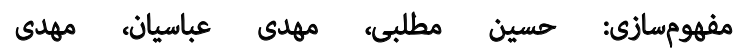

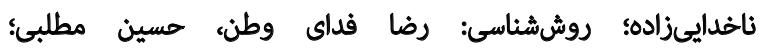

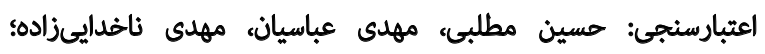

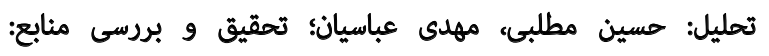

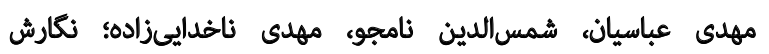

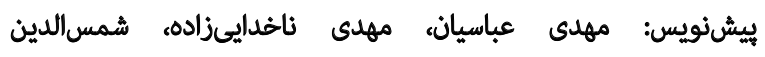

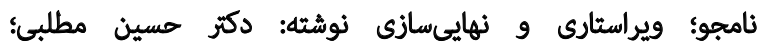
بصرىسازى و نظارت: حسين مطلبى، رضا فداى وطني؛ مديريت

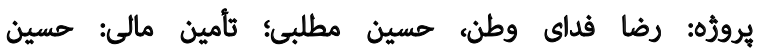
مطلبى، مهدى عباسيان. 


\section{References}

[1] United Nations, Department of Economic and Social Affairs, Population Division. World population ageing 2013 Geneva: United Nations; 2013. [DOI:10.1787/eco_surveys-bel2013-graph13-en]

[2] Moshfeq M, Husseini Q. [Futurology of Iran's demographic changes from 1390 to 1420 (2011-2041) (Persian)]. Ma'rifat-i Farhangi Ejtemaii. 2012; 4(1):21-42.

[3] Barnett I, van Sluijs EM, Ogilvie D. Physical activity and transitioning to retirement: A systematic review. American Journal of Preventive Medicine. 2012; 43(3):329-36. [DOI:10.1016/j.amepre.2012.05.026]

[4] Moti'e Haghshenas N. [Sociological dimension of the challenges of population aging and active aging in Iran (Persian)]. Quarterly Journal of Sociological Studies of Iran. 2011; 1(2):133-47.

[5] Tomiak M, Berthelot JM, Guimond E, Mustard CA. Factors associated with nursing-home entry for elders in Manitoba, Canada. The Journals of Gerontology Series A: Biological Sciences and Medical Sciences. 2000; 55(5):M279-87. [DOI:10.1093/ gerona/55.5.M279]

[6] National Library of Medicine. Nursing homes [Internet]. 2016 [Updated 2016 September 12]. Available from: https://www. nlm.nih.gov/medlineplus/nursinghomes.html

[7] Shojaei M, Maghsoudnia Sh. [Gerontology and geriatrics (Persian)]. Tehran: Janbazan Medical and Engineering Research Center and Research Center of Elderly Health and Social Issues; 2005.

[8] Family Caregiver Alliance, National Center on Caregiving. Selected long-term care statistics [Internet]. 2016 [updated 2016 September 12]. Available from: https://www.caregiver.org/ selected-long-term-care-statistics

[9] Willink A, Davis K, Schoen C. Risks for nursing home placement and medicaid entry among older medicare beneficiaries with physical or cognitive impairment. Issue Briefs, Commonwealth Fund; 2016; 37:1-14.

[10] Salarvand S, Abedi HA. [Causes and motivations of elderly home residency from residents' point of view (Persian)]. FEYZ Journal of Kashan University of Medical Sciences. 2008; 12(2):55-61.

[11] Lee DT. Transition to residential care: experiences of elderly Chinese people in Hong Kong. Journal of Advanced Nursing. 1999; 30(5):1118-26. [DOI:10.1046/j.1365-2648.1999.01196.x]

[12] Waldrop DP, Meeker MA. Crisis in caregiving: When homebased end-of-life care is no longer possible. Journal of Palliative Care. 2011; 27(2):117-25. [DOI:10.1177/082585971102700207]

[13] Heravi Karimloo M, Anoosheh M, Foroughan M, Sheykhi MT, Hajizade E, Seyed Bagher Maddah MS, et al. [Loneliness from the perspectives of elderly people: A phenomenological study (Persian)]. Iranian Journal of Ageing. 2008; 2(4):410-20.

[14] Shiri T, Azimi N. [A comparative study of qualitative content analysis and hermeneutics (Persian)]. Journal of Sociology Studies. 2012; 5(15):79-99.

[15] Matlabi H, Hamedi Behtash H, Shafiei M. [Admission to a nursing home: Viewpoints of institutionalized older people about replacement (Persian)]. Elderly Health Journal. 2016; 2(1):1-5.
[16] Matlabi H. [The contribution of home-based technology to elderly people's quality of life in UK-specific extra care housing (Persian)]. Paper presented at: The $1^{\text {st }}$ International $\& 4^{\text {th }}$ National Congress on Health Education \& Promotion; 16-19 May 2011; Tabriz, Iran.

[17] Scocco P, Rapattoni M, Fantoni G. Nursing home institutionalization: a source of eustress or distress for the elderly. International Journal of Geriatric Psychiatry. 2006; 21(3):281-7. [DOI:10.1002/ gps.1453]

[18] Gaugler JE, Duval S, Anderson KA, Kane RL. Predicting nursing home admission in the US: A meta-analysis. BMC Geriatrics. 2007; 7(1):1. [DOI:10.1186/1471-2318-7-13]

[19] Greene VL, Ondrich JI. Risk factors for nursing home admissions and exits: A discrete-time hazard function approach Journal of Gerontology. 1990; 45(6):S250-8. [DOI:10.1093/ geronj/45.6.S250] [PMID]

[20] Szebehely M, Trydegård GB. Home care for older people in Sweden: A universal model in transition. Health \& Social Care in the Community. 2012; 20(3):300-9. [DOI:10.1111/j.13652524.2011.01046.x]

[21] Caro FG, Porell FW, Sullivan DM, Safran-Norton CE, Miltiades $\mathrm{H}$. Home health and home care in Massachusetts after the Balanced Budget Act of 1997: Implications of cost containment pressures for service authorizations. Home Health Care Services Quarterly. 2002; 21(1):47-66. [DOI:10.1300/J027v21n01_03]

[22] Luppa M, Luck T, Weyerer S, König HH, Brähler E, RiedelHeller SG. Prediction of institutionalization in the elderly: A systematic review. Age and Ageing. 2009; 39(1):31-8. [DOI:10.1093/ ageing/afp202] [PMID] 
This Page Intentionally Left Blank 